\title{
System Identifiability from Finite Time Series*
}

\author{
CHRISTIAAN HEIJ $\dagger$ \\ The possibility to identify linear systems from finite data, e.g. a partial \\ impulse response, is determined by the degree of corroboration of system \\ restrictions by the observed data.
}

Key Words-System identification; identifiability; linear systems; time-series analysis; corroboration; partial realization theory.

\begin{abstract}
In this paper we investigate the identification of systems from time series observed over a finite time interval. The data generating system is supposed to be finite dimensional, linear and time invariant, but not necessarily controllable. The minimal number of time series needed to identify a system is characterized by the identifiability index of a system, which measures the rank drop of autoregressive representations. We formulate a procedure for modelling finite time series which takes the corroboration of system restrictions into account. This also gives a new solution for the partial realization problem.
\end{abstract}

\section{INTRODUCTION}

Stated in General terms, identifiability concerns the question whether the information contained in the available data on an object suffices to determine or identify that object uniquely. The investigation of identifiability requires a specification of the available information, of the characteristics of interest, and of the method to infer characteristics from the data. Within the context of dynamical systems mainly two specifications have received attention, which we denote by parameter identifiability and system identifiability.

The parameter identifiability problem has received most attention in statistics, econometrics and systems theory. The main question is whether the model parameters can be uniquely deduced from the available information. In the case of stochastic dynamical systems this information is of a probabilistic nature, e.g. the spectrum of the process. For deterministic systems the information consists of functional relationships, e.g. the transfer function. A

\footnotetext{
Received 2 October 1991; revised 1 April 1992; reviscd 14 September 1992; received in final form 22 November 1992. The original version of this paper was not presented at any IFAC meeting. This paper was recommended for publication in revised form by Associate Editor $T$. Başar under the direction of Editor H. Kwakernaak. Corresponding author Heij's Telephone + 31-10-4081269; Fax +31-10-4527746.

† Econometric Institute, Erasmus University Rotterdam, P.O. Box 1738, 3000 DR Rotterdam, The Netherlands.
}

special case is the so-called realization problem, which concerns the deduction of the parameters of a state space model from the spectrum or the transfer function. There is an abundant literature on parameter identifiability and the related issue of canonical forms, see e.g. Fisher (1966), Hsiao (1983), Gevers and Wertz (1984, 1987), Hannan and Deistler (1988), and the references therein.

Parameter identifiability can be seen as a matter of deduction of the internal model parameters from complete information on the external system characteristics. System identifiability is more a matter of perfect induction. It concerns the question of whether the external system behaviour can be exactly estimated from observed data generated by the system. The answer will depend on the information content in the data and on the method of inference, i.e. the identification procedure for modelling the observed time series. Some experiments are particularly informative. For example, the impulse response gives a complete characterization of the transfer function for linear, time invariant and controllable input-output systems. This requires that the response is measured over an infinitely long time interval. As another example, the response to sinusoidal inputs also characterizes the system, provided that the model order is known and that the frequency of the sinusoid is varied sufficiently often. The system identifiability problem from arbitrary exact data of deterministic controllable inputoutput systems has been investigated by, among others, Grewal and Glover (1976), Sontag (1979, 1980), Kalman (1983) and Chen (1987). A closely related question for stochastic systems is that of consistency, i.e. the question of whether the generating system can be identified in the limit if the number of observations tends to infinity, cf. Ljung (1987) and Caines (1988), and the references therein. 
In this paper we investigate system identifiability from arbitrary exact data without assuming any prior information on the inputoutput structure or the controllability of the system. We consider a system as a set of admissible trajectories for the external variables under consideration. This system concept in terms of the external behaviour was introduced in Willems $(1986 \mathrm{a}, \mathrm{b}, 1987,1991)$. It has the advantage that system concepts and properties can be analysed directly by means of the behaviour, that is, independent of the chosen system representation such as polynomial descriptions, transfer functions, or state space models.

The assumed available information on the system consists of observed data and structural information. We suppose that the data consist of a finite number of multivariate time series observed over finite time intervals. The structural information consists of qualitative properties of the data generating system, i.e. that it is linear, time invariant, and complete in the sense that it can be specified by local restrictions. No information is assumed concerning quantitative properties like the number of input and output variables, the number and initial conditions of state variables, or the observabiilty indices. The system also need not be controllable.

The central question is whether observation of finite time series generated by a linear, time invariant, complete system allows us to infer the full external system behaviour, i.e. the set of all time series which are compatible with this system. Hereby it is assumed that the data are exact, i.e. the time series are indeed generated by a linear, time invariant, complete system and they are not disturbed in any way. This system identifiability problem resembles that investigated by Guidorzi $(1975,1981)$. A main issue is that the computation of the system from observed data requires that all the noncontrollable modes must be excited (Guidorzi (1981)). As our analysis will show, if only a single realization of a non-controllable system is observed it may be impossible to fulfil this state excitation condition. The so-called identifiability index of a system describes the minimally required number of experiments needed for the identification of the non-controllable part of the system. Further, in the case of finite time series there may arise an incompatibility of the regularities exhibited in the data obtained from the different experiments. The present paper analyses these problems and proposes an identification procedure which takes these aspects into account. The data generating system can be recovered by this procedure if sufficient experiments have been performed for a sufficiently long time.

The main result of this paper is the definition and analysis of a procedure for modelling a finite number of observed finite time series (Section 4) which has attractive identifiability properties (Section 5). It also provides an alternative solution for the partial realization problem (Section 6). The procedure is based on principles of falsification and corroboration. First we determine the collection of all relationships which are unfalsified by the data, and then some or all of these relationships may be eliminated if they are not sufficiently corroborated. The resulting procedure has the property that more precise systems are identified if the observation interval is increased. System identifiability is characterized in terms of the identifiability index. This measures the rankdrop of an autoregressive representation of the system, that is, the difference between the maximally and minimally achievable rank of the system representation.

In order to describe the procedure and the identifiability results we first present some preliminary concepts and results (Sections 2 and 3). This paper on identifiability from data consisting of multiple finite time series completes corresponding results for infinite time series in Heij (1992) and for a single finite time series in Heij $(1988,1992)$.

\section{PRELIMINARY CONCEPTS}

In this section we summarize some concepts related to systems and identifiability which are more thoroughly exposed in, respectively, Willems (1986a, b, 1987, 1991) and Heij (1992).

Assume that the data concerns $q$ real-valued variables observed in discrete time. A dynamical system is defined as a subset $\mathscr{B}$ of the set of all $q$-dimensional time series, i.e. $\mathscr{B} \subset\left(\mathbb{R}^{q}\right)^{Z}$. The structural information on the observed system consists of the qualitative properties of linearity, time invariance, and completeness. This means that $\mathscr{B}$ is a linear and shift invariant set which imposes only local restrictions on the data (in the sense that a time series $w$ belongs to $\mathscr{B}$ if and only if $\left.\left.w\right|_{T} \in \mathscr{B}\right|_{T}$ for all finite time sets $T \subset \mathbb{Z}$ ).

A system is called controllable if every past can be driven into any future (in the sense that for all $w_{1}, w_{2} \in \mathscr{B}$ and $t_{1}, t_{2} \in \mathbb{Z}$ there exist $w \in \mathscr{B}$ and $n \in \mathbb{N}$ such that $\left.w\right|_{\left(-\infty, r_{1}\right]}=\left.w_{1}\right|_{\left(-\infty, t_{1}\right]}$ and $\left.\left.w\right|_{\left.t_{1}+n, \infty\right)}=\left.w_{2}\right|_{\left(t_{2}, \infty\right)}\right)$. A system is called autonomous if the past uniquely implies its future (i.e. if $w_{1}, w_{2} \in \mathscr{B}$ and $t \in \mathbb{Z}$ with $\left.w_{1}\right|_{(-x, t)}=$ $\left.w_{2}\right|_{(-\infty, t]}$, then $\left.w_{1}=w_{2}\right)$. 
Let $\mathbb{B}$ denote the class of linear, time invariant, complete systems. Such systems have autoregressive representations, expressing a system as the solution set of a number of equations $\sum_{k=k_{1}}^{k_{2}} R_{k} w(t+k)=0, t \in \mathbb{Z}$, with $R_{k} g \times q$ real matrices for some $g \in \mathbb{N}$. This is denoted by $\mathscr{B}=\mathscr{B}(R):=\left\{w \in\left(\mathbb{P}^{4}\right)^{2} ; \quad R\left(\sigma, \sigma^{-1}\right) w=0\right\}$, where $\sigma$ denotes left shift and $R \in \mathbb{R}^{g \times 4}\left[s, s^{-1}\right]$ denotes the polynomial matrix $R=\sum_{k=k_{1}}^{k_{2}} R_{k} s^{k}$. The representation of a system $\mathscr{B} \in \mathbb{B}$ by autoregressive laws is highly non-unique. A minimal representation contains as few equations as possible and the equations are of lowest possible degree. Here the degree of $r=$ $\sum_{k=k_{1}}^{k_{2}} r_{k} s^{k} \in \mathbb{R}^{1 \times q}\left[s, s^{-1}\right]$ is defined as $\max \left\{k ; r_{k} \neq 0\right\}-\min \left\{k ; r_{k} \neq 0\right\}$. The degrees of a minimal representation coincide with the (Kronecker) system observability indices $v_{1} \geq$ $\cdots \geq v_{p} \geq 0$. The minimal number $p$ of equations gives the number of output variables of the system, the number of input variables is $m:=q-p$, and $n:=\sum_{i=1}^{p} v_{i}$ is the number of state variables in a minimal realization of the system.

Let the data consist of $N$ time series $w_{i} \in\left(\mathbb{R}^{q}\right)^{\mathbb{Z}}$ observed on the time interval $T_{i} \subset \mathbb{Z}, i \in[1, N]$, so that $d=\left\{\left.w_{i}\right|_{r} ; i \in[1, N]\right\}$. Let $D$ denote any subset of the set of all finite numbers of observed time series of finite or infinite length. An identification procedure specifies which systems are identified for the observed data. In this paper we will require that the identified system is unique and belongs to $\mathbb{B}$, so that the procedure is a mapping $P: D \rightarrow \mathbb{B}$.

Our procedures are based on the autoregressive laws satisfied by the data. A law $r \in \mathbb{R}^{1 \times q}\left[s, s^{-1}\right]$ is called unfalsified by the data if $\left.\left.w_{i}\right|_{T_{i}} \in \mathscr{B}(r)\right|_{T_{1}}$ for all $i \in[1, N]$. A collection of laws $L \subset \mathbb{R}^{1 \times q}\left[s, s^{-1}\right]$ is called compatible with the data if the conjunction of all laws in $L$ is unfalsified (i.e. $\left.\left.w_{i}\right|_{T_{t}} \in(\cap\{\mathscr{B}(r) ; r \in L\})\right|_{T}$, for all $i \in[1, N])$.

Further we use a generalization of the algebraic genericity concept for finite dimensional spaces. A set $G \subset\left(\left(\mathbb{R}^{q}\right)^{\mathbb{Z}}\right)^{N}$ is called $\mathscr{B}$-generic if its complement is small in the sense that $\left(\mathscr{B}^{N} \backslash G\right) \subset \bigcup_{n \in \mathbb{N}}\left\{p_{n}^{-1}(0) ; n \in N\right\}$, where $p_{n}:\left(\left(\mathbb{R}^{q}\right)^{\mathbb{Z}}\right)^{N} \rightarrow \mathbb{R}$ are non-zero polynomials.

Definition 2.1. A system $\mathscr{B}$ is called $(N, T)$ identifiable by a procedure $P$ if for observed data $d=\left\{\left.w_{i}\right|_{T_{i}} ; i \in[1, N]\right\}, \quad T=\left\{T_{1}, \ldots, T_{N}\right\}$, there holds $P(d)=\mathscr{B}$ for generic $\left\{w_{1}, \ldots, w_{N}\right\} \in \mathscr{B}^{N}$.

Hence a system is identifiable if it can be identified on the basis of generic observed data. It can be shown that for the procedures considered in this paper the identifiability condition is equivalent to the existence of some (as opposed to generic) data $d$ such that $P(d)=\mathscr{B}$. This equivalence is essentially similar to that for real-valued polynomial functions, i.e. such a function is generically non-zero if and only if it is somewhere non-zero.

The following corroboration concept reflects the idea that there can be no evidence from observed data for laws which are of a type which are generically also unfalsified for observations from the lawless system $\mathscr{B}=\left(\mathbb{R}^{4}\right)^{\mathbb{Z}}$.

\section{Definition 2.2.}

(i) If for $\left(\mathbb{R}^{q}\right)^{2}$-generic data observed on $T=\left\{T_{1}, \ldots, T_{N}\right\}$ there exists an unfalsified law of degree $\delta$, then laws of this degree are uncorroborated.

(ii) A law is called corroborated by data on $T$ if it is unfalsified and not uncorroborated.

In order to clarify these somewhat abstract notions we give two simple examples.

Example 1. As a first example we consider the univariate system described by $\mathscr{B}=\mathscr{B}(\sigma-1)=$ $\left\{w \in \mathbb{R}^{\mathbb{Z}} ; w(t+1)=w(t)\right.$ for all $\left.t \in \mathbb{Z}\right\}$, that is, the system consisting of constant time series.

First consider the case $N=1$ of one time series observed on the interval $T=[1, \tau]$. If $\tau=1$, then $\left.\mathscr{B}\right|_{T}=\mathbb{R}$ and of course no regularity can be detected. If $\tau=2$, then the system behaviour $\left.\mathscr{B}\right|_{T}$ is non-generic in the observation space $\mathbb{R}^{2}$, as $\left\{w \in \mathbb{R}^{Z} ;\left.\left.w\right|_{T} \in \mathscr{B}\right|_{T}\right\}=p^{-1}(0)$ where $p(w):=$ $w(2)-w(1)$. However, this system behaviour is not fully observed and only a single realization of it is available. Note that for generic data observed on $T=[1,2]$ there exists an unfalsified law of degree one. Indeed, for $\left.w\right|_{T} \in \mathbb{B}^{2}$ with $w(1) \neq 0$ there holds $w(2)=\beta w(1)$, where $\beta=w(2) / w(1)$. For observations from $\mathscr{B}$ we obtain $\beta=1$, but this is no more surprising than finding any other value of $\beta$ for observations from other systems than $\mathscr{B}$. This means that laws of degree one are not corroborated for such a short observation interval. Now suppose that $\tau \geq 3$. For $w \in \mathbb{R}^{\mathbb{Z}}$ define $p(w):=\operatorname{det}\left(H_{1}\right)$, where $H_{1}$ is the Hankel matrix

$$
H_{1}=\left(\begin{array}{ll}
w(1) & w(2) \\
w(2) & w(3)
\end{array}\right)
$$

Generically on $\mathbb{R}^{\mathbb{Z}}$ there does not exist an unfalsified law of degree one, as this would require that $p(w)=w(1) w(3)-\{w(2)\}^{2}=0$. As for observations $\left.\left.w\right|_{r} \in \mathscr{B}\right|_{r}$ such a law is indeed unfalsified, this means that the system law $\sigma-1$ is corroborated by data from $\left.\mathscr{B}\right|_{r}$ for $\tau \geq 3$.

Finally consider the case of $N=2$ observations $w_{i}$ on intervals $T_{i}, i=1,2$. If $\tau_{1}=\tau_{2}=1$ then no regularities can be detected. If, e.g. $\tau_{1}=\tau_{2}=2$ 
then the data show an exceptional regularity, as they satisfy det $\left(\mathrm{H}_{2}\right)=0$ where

$$
H_{2}=\left(\begin{array}{ll}
w_{1}(1) & w_{2}(1) \\
w_{1}(2) & w_{2}(2)
\end{array}\right) .
$$

Such a relationship is non-generic in the observation space $\mathbb{R}^{2} \times \mathbb{R}^{2}$, so that the law $(\sigma-1) w_{i}=0, i=1,2$, is corroborated.

These results suggest certain identifiability properties of the system $\mathscr{B}$. General results are obtained in, e.g. Theorem 5.2 and Proposition 5.6 of this paper.

Example 2. Our second example is related to deterministic realization theory. We consider data consisting of the (causal) impulse response of a linear system. Suppose that the input $u$ and the output $y$ of the system are related according to $y(t)=y(t-4)+u(t-1)+u(t-2)+u(t-3)$. If the input consists of an impulse, i.e. $u(0)=1$ and $u(t)=0$ for $t \neq 0$, then the corresponding (causal) output response on the time interval $[1, \infty)$ is given by

$$
y=\left(\begin{array}{lllllllllllll}
1 & 1 & 1 & 0 & 1 & 1 & 1 & 0 & 1 & 1 & 1 & 0 & \cdots
\end{array}\right) .
$$

The available data consist of $y$ observed on the partial interval $T=[1, \tau]$. Unfalsified laws correspond to linear dependencies between the rows of the Hankel matrix

$$
H=\left(\begin{array}{ccccccc}
1 & 1 & 1 & 0 & 1 & 1 & \cdots \\
1 & 1 & 0 & 1 & 1 & 1 & \cdots \\
1 & 0 & 1 & 1 & 1 & 0 & \cdots \\
\vdots & \vdots & \vdots & \vdots & \vdots & \vdots &
\end{array}\right) .
$$

A law of degree $\delta$ corresponds to the linear dependency of row $\delta+1$ in $H$ on the foregoing ones. For the partial observation such a dependency can only be checked in the left top corner of $H$, and this involves a matrix of size $(\delta+1) \times(\tau-\delta)$. Such a row dependency is generic if the number of columns is less than the number of rows. So an unfalisfied law is corroborated if and only if $\tau-\delta \geq \delta+1$, i.e. if $\delta \leq(\tau-1) / 2$.

If $\tau=1$ or $\tau=2$ then there are no corroborated laws. For $\tau=3$ the law $(\sigma-1) y=$ 0 is corroborated. It is easily checked that for $4 \leq \tau \leq 8$ there are no corroborated laws. For $\tau=9$ the leading $5 \times 5$ submatrix of $H$ has rank 4 , as the fifth row is equal to the first one. The corresponding law $\left(\sigma^{4}-1\right) y=0$ is corroborated. The same holds true for $\tau \geq 10$.

This indicates certain implications for the partial realization problem. For example, if $\tau=5$ then the partial Hankel matrix has rank 3 and there exists a third order law, e.g. $\left(\sigma^{3}+\sigma^{2}-\right.$ 1) $y=0$. However, there is no reason to conclude from this that the impulse response is generated by a third order system. Indeed. for systems of every state dimension the partial impulse response on $T=[1,5]$ will generically satisfy a third order law. On the other hand, the fourth order law on $T=[1,9]$ is not generic. So there is then evidence that the data come from a fourth order system.

In modelling the impulse response it would of course make more sense to take also the input into account. The corresponding partial realization problem is further discussed in Section 6 .

\section{PRELIMINARY RESULTS}

In this section we summarize some identifiability results for data consisting either of infinite time series or of a single finite time series. For details and illustrations we refer to Heij (1992).

First we consider the case where the data consist of a number $N$ of time series given over the full time set $\mathbb{Z}$. so that $T=\mathbb{Z}^{N}$ and $d=\left\{w_{1}, \ldots, w_{N}\right\}$ with $w_{i} \in\left(\mathbb{R}^{q}\right)^{\mathbb{Z}}, i \in[1, N]$.

Proposition 3.1. For data on $T=\mathbb{Z}^{N}$ every unfalsified law is corroborated and the collection of all unfalsified laws is compatible with the data.

For a polynomial matrix $R \in \mathbb{R}^{g \times 4}\left[s, s^{-1}\right]$ define the rank drop as

$$
\begin{aligned}
I(R):= & \max \left\{\operatorname{rank}\left(R\left(s, s^{-1}\right)\right) ; 0 \neq s \in \mathbb{C}\right\} \\
& -\min \left\{\operatorname{rank}\left(R\left(s, s^{-1}\right)\right) ; 0 \neq s \in \mathbb{C}\right\},
\end{aligned}
$$

and for $\mathscr{B}=\mathscr{B}(R)$ define the identifiability index as $I(\mathscr{B}):=\max \{1, I(R)\}$. This index is welldefined, i.e. independent of the choice of the representation $R$ of the system $\mathscr{B}$. The next result describes identifiability properties of the procedure of most powerful unfalsified modelling, defined by $P^{*}(d):=\bigcap\{\mathscr{B}(r) ; r$ unfalsified by $d\}$.

Theorem 3.2. A system $\mathscr{B} \in \mathbb{B}$ is $\left(N, \mathbb{Z}^{N}\right)$ identifiable by $P^{*}$ if and only if $N \geq I(\mathscr{B})$.

Next we consider the case where the data consist of a single multivariate time series observed on a finite time interval $T$ of length $\tau$, i.e. $d=\left\{\left.w\right|_{T}\right\}$ for some $w \in\left(\mathbb{R}^{q}\right)^{Z}$.

Proposition 3.3. For data consisting of one finite time series observed on an interval of length $\tau$ there holds:

(i) an unfalsified law is corroborated if and only if it has degree $\delta \leq(\tau-q) /(q+1)$;

(ii) for most systems $\mathscr{B} \in \mathbb{B}$ the collection of all unfalsified laws is $\mathscr{B}$-generically not compatible with the data; 
(iii) for every system $\mathscr{B} \in \mathbb{B}$ the collection of all corroborated laws is $\mathscr{B}$-generically compatible with the data.

Define $P_{1}^{*}$ as the procedure which accepts all corroborated laws provided that they are compatible with the data, i.e. provided that the system which satisfies all corroborated laws is unfalsified by the data. Formally, let $\mathscr{B}^{*}(d):=$ $\cap\{\mathscr{B}(r) ; r$ corroborated by $d\}$, then $P_{1}^{*}(d)=$ $\mathscr{B}^{*}(d) \quad$ if $\left.\quad d \in \mathscr{B}^{*}(d)\right|_{T} \quad$ and $\quad P_{1}^{*}(d)=\left(\mathbb{R}^{q}\right)^{\mathbb{Z}}$ otherwise.

Theorem 3.4. A system $\mathscr{B} \in \mathbb{B}$ is $(1, T)$ identifiable by $P_{1}^{*}$ if and only if $I(\mathscr{B})=1$ and the length of the observation interval is $\tau \geq\left(v_{1}+1\right)(q+$ 1) -1 , where $v_{1}$ is the largest observability index of $\mathscr{B}$.

Example 3. We illustrate the foregoing results by considering again the partial impulse response data as in Example 2. There we showed that corroboration requires that the number of columns in a corresponding partial Hankel matrix is not less than the number of rows. For a single $q$-variate time series and a law of degree $\delta$ this partial matrix has size $q(\delta+1) \times(\tau-\delta)$. Corroboration then requires that $\tau-\delta \geq q(\delta+$ 1), which shows Proposition 3.3 (i).

If, e.g. $\tau=11$ then it is easily checked that the collection of all unfalsified laws is not compatible with the data. Not only the law $\left(\sigma^{4}-1\right) y=0$ is unfalsified, but also, e.g. the law $\left(\sigma^{9}-1\right) y=0$. The conjunction of these two laws would require that $(\sigma-1) y=0$ which is of course falsified. On the other hand, the collection of all corroborated laws is compatible with the data. It is easily checked from the leading $6 \times 6$ partial Hankel matrix that this class of laws is given by $r(\alpha, \beta):=(\alpha \sigma+\beta)\left(\sigma^{4}-1\right), \alpha, \beta \in \mathbb{R}$. The conjunction of all these laws gives the system $\bigcap\{\mathscr{B}(r(\alpha, \beta)) ; \alpha, \beta \in \mathbb{R}\}=\mathscr{B}\left(\sigma^{4}-1\right)$ which is unfalsified by the data. Similar results hold true for other values of the length $\tau$ of the observation interval.

For these data the law $\left(\sigma^{4}-1\right) y=0$ which holds true for the full impulse response is identified by the procedure $P_{1}^{*}$ for $\tau \geq 9$. This illustrates Theorem 3.4 , as in this case $l=1$, $q=1$ and $v_{1}=4$.

\section{IDENTIFICATION PROCEDURE FOR MULTIPLE OBSERVED FINITE TIME SERIES}

In this section we formulate a procedure for modelling a finite number of observed finite time series. We use the following notation. Let the data consist of $d=\left\{\left.w_{i}\right|_{T_{i}} ; i \in[1, N]\right\}$, where $N \in \mathbb{N}$ denotes the number of time series and where $w_{i} \in\left(\mathbb{R}^{q}\right)^{\mathbb{Z}}$ is observed on an interval $T_{i} \subset \mathbb{Z}$ of finite length $\tau_{i}, i \in[1, N]$. We order the observations so that $\tau_{1} \geq \tau_{2} \geq \cdots \geq \tau_{N} \geq 1$. Let $T=\left\{T_{1}, \ldots, T_{N}\right\}$ and let

$$
\delta^{*}(T):=\max \left\{\delta ; \sum_{i=1}^{N}\left[\tau_{i}-\delta\right]^{+} \geq q(\delta+1)\right\},
$$

where $\left[\tau_{i}-\delta\right]^{+}:=\max \left\{\tau_{i}-\delta, 0\right\}$.

The following proposition expresses the main respect in which the case of multiple observed finite time series differs from that of a single observed finite time series, cf. Proposition 3.3.

Proposition 4.1. For data consisting of multiple observed finite time series, i.e. with $N>1$, there holds that

(i) an unfalsified law is corroborated if and only if it has degree $\delta \leq \delta^{*}(T)$;

(ii) the collection of all corroborated laws need not be generically compatible with the data, i.e. there exist systems $\mathscr{B} \in \mathbb{B}$ and $(N, T)$ for which this collection is $\mathscr{B}$-generically not compatible.

\section{Proof. See Appendix.}

Example 4. The main idea is that, e.g. one relatively long time series may lead to a large collection of laws of high degree which are corroborated by this time series, while this collection implies laws of low degree which are falsified by other time series observed on shorter time intervals.

We illustrate this by a simple example. Consider the system in $q=3$ variables described by $\mathscr{B}=\left\{w \in\left(\mathbb{R}^{3}\right)^{\mathbb{Z}} ;(\sigma-1) w=0\right\}=\left\{w \in\left(\mathbb{R}^{3}\right)^{\mathbb{Z}} ;\right.$ $\exists c \in \mathbb{R}^{3}$ such that $\left.w(t)=c, \forall t \in \mathbb{Z}\right\}$. Let the data consist of $N=2$ observations $d=\left\{\left.w_{1}\right|_{T_{1}},\left.w_{2}\right|_{T_{2}}\right\}$, so that $w_{i}(t)=c_{i}, t \in \mathbb{Z}$, for some $c_{i} \in \mathbb{R}^{3}, i=1,2$. Let $K_{i}:=\operatorname{ker}\left(c_{i}\right), \quad i=1,2$, then there holds $\mathscr{B}$-generically that $\operatorname{dim}\left(K_{1}\right)=2$ and $\operatorname{dim}\left(K_{1} \cap\right.$ $\left.K_{2}\right)=1$. To be explicit, $\left\{\left(w_{1}, w_{2}\right) \in \mathscr{B}^{2}\right.$; $\left.\operatorname{dim}\left(K_{1} \cap K_{2}\right) \neq 1\right\}=\left\{\left(w_{1}, w_{2}\right) \in \mathscr{B}^{2} ; c_{1}=0\right.$ or $c_{2}=0$ or $\left.c_{1}=c_{2}\right\}=p^{-1}(0)$ where $p\left(w_{1}, w_{2}\right):=$ $q\left(w_{1}\right) q\left(w_{2}\right) q\left(w_{1}-w_{2}\right)$ with $q(w):=w(0)^{T} w(0)$. Evidently, $p$ is not identically zero on $\mathscr{B}^{2}$. Suppose that the data are generic in this sense, so that there exists an $a \in K_{1} \backslash K_{2}$. Then the static law $a^{T} w=0$ is satisfied by the first observation but not by the second one. Now suppose that the first observation interval is much longer than the second one, in particular that there exists $\delta \in \mathbb{N}$ for which $\tau_{2} \leq \delta \leq\left(\tau_{1}-3\right) / 4$. This means that laws of degree $\delta$ are unfalsified (and uncorroborated) by the second observation. Further, 
for every $r \in \mathbb{R}\left[s, s^{-1}\right]$ of degree $\delta$ the law $r \cdot a^{T}$ is corroborated by the first observation, as it is of course unfalsified and it has degree $\delta \leq \delta^{*}(T)=$ $\max \left\{\delta ; \tau_{1}-\delta \geq 3(\delta+1)\right\}=\left(\tau_{1}-3\right) / 4$. Although every such law is individually corroborated by the data, the collection of these laws $\left\{r \cdot a^{r} ; d(r)=\delta\right\}$ is not compatible with the data. Indeed, the system which satisfies all these laws is given by $\cap\left\{\mathscr{B}\left(r \cdot a^{T}\right) ; d(r)=\delta\right\}=$ $\mathscr{B}\left(a^{T}\right)$, and $w_{2} \notin \mathscr{B}\left(a^{T}\right)$ as $a^{T} c_{2} \neq 0$. This implies that also the collection of all corroborated laws is not compatible with the data.

We mention that for controllable systems such problems generically do not occur. Controllable systems have identifiability index one, so that according to Theorem 3.4 the system is identified from generic observations on a sufficiently long time interval. The corroborated laws then consist of system laws, so that falsification by short time series cannot take place.

As the corroborated laws need not be compatible we have to decide which laws should be accepted and which should be rejected. It seems attractive to accept laws with a large degree of corroboration, defined as the number of times a law is independently verified by the data. Note that a law of degree $\delta$ can be verified by the $i$ th observation if and only if $\tau_{i} \geq \delta+1$, in which case the law implies $\tau_{i}-\delta$ independent restrictions.

Definition 4.2. A corroborated law of degree $\delta$ has a degree of corroboration $C_{\delta}:=\sum_{i=1}^{N}\left[\tau_{i}-\right.$ $\delta\}^{+}$.

The following procedure $P_{f}^{*}$ accepts the most strongly corroborated laws. More precisely, corroborated laws of a certain degree of corroboration are accepted if and only if they are compatible with the collection of laws with a larger degree of corroboration.

Definition 4.3. For data $d$ consisting of multiple observed finite time series let $\mathscr{R}_{\delta}$ denote the set of all unfalsified laws of degree at most $\delta$ and let $\mathscr{R}^{*}=\mathscr{R}_{\delta}$. with $\delta^{*}:=\max \left\{\delta \in\left[0, \delta^{*}(T)\right] ; \mathscr{R}_{\delta}\right.$ compatible with $d\}$. Then $P_{f}^{*}$ accepts the laws of $\mathscr{R}^{*}$, i.e. $P_{f}^{*}(d):=\bigcap\left\{\mathscr{R}(r) ; r \in \mathscr{R}^{*}\right\}$.

In the case of a single observation this procedure coincides for generic data with the procedure $P_{1}^{*}$ defined in Section 3 , cf. Proposition 3.3 (iii). The identified system $P_{f}^{*}(d)$ can be determined by checking row depend- encies in (partial) Hankel matrices defined in terms of the observed data. We illustrate this by means of a simple example and then give an outline of the general algorithm.

Example 5. Consider the same system and data as presented in Example 4, with observation intervals $T_{i}=\left[1, \tau_{i}\right], i=1,2, \tau_{1} \geq \tau_{2}$.

First we suppose that $\tau_{1}=5$ and $\tau_{2}=3$. In this case we analyse the following partial Hankel matrix $\left(a^{*}\right.$ denotes an unobserved element).

$$
\begin{aligned}
& w_{1}(1) \quad w_{2}(1) w_{1}(2) \quad w_{2}(2) \quad w_{1}(3) \quad w_{2}(3) \quad w_{1}(4) * w_{1}(5) * \\
& w_{1}(2) w_{2}(2) w_{1}(3) w_{2}(3) w_{1}(4) * w_{1}(5) * * * \\
& w_{1}(3) w_{2}(3) w_{1}(4) * w_{1}(5) * * * * * \\
& w_{1}(4) * w_{1}(5) * * * * * * \\
& w_{1}(5) * * * * * * * * * *
\end{aligned}
$$

As before, let $w_{i}(t)=c_{i} \neq 0$ and $K_{i}=\operatorname{ker}\left(c_{i}^{T}\right)$, $i=1,2$, with $\operatorname{dim}\left(K_{1} \cap K_{2}\right)=1$, and let $0 \neq$ $a_{0} \in K_{1} \cap K_{2}$. Then $r_{0}=a_{0}^{T}$ is the only unfalsified law of degree 0 . This is checked by determining the left kernel of the first block-row with dimension $3 \times 8$. Unfalsified laws of degree 1 are obtained from the left kernel of the matrix

$$
\left(\begin{array}{llllll}
w_{1}(1) & w_{2}(1) & w_{1}(2) & w_{2}(2) & w_{1}(3) & w_{1}(4) \\
w_{1}(2) & w_{2}(2) & w_{1}(3) & w_{2}(3) & w_{1}(4) & w_{1}(5)
\end{array}\right) .
$$

Note that this matrix has dimension $6 \times 6$, so that a row-rank deficiency is non-generic. This is reflected by the corroboration condition $\delta \leq$ $\delta^{*}(T)=1$. A basis for the left kernel corresponds to the autoregressive laws $(\sigma-1) w=0$ and $\left(\alpha a_{0}^{T}+\beta a_{0}^{T} \sigma\right) w=0$. These corroborated laws are compatible with the data, and laws of degree larger than one are not corroborated. Hence in this case the identified system is $\mathscr{B} \cap \mathscr{B}\left(a_{0}^{T}\right)$.

Next suppose that $\tau_{1}=11$ and $\tau_{2}=2$, so that $\delta^{*}(T)=2$. The corroborated laws of degrees 0 and 1 are as before. Additional corroborated laws of degree 2 are given by

$$
\left(\sigma^{2}-\sigma-\left(\begin{array}{l}
1 \\
1 \\
1
\end{array}\right) a_{1}^{T}\right) w=0,
$$

where $a_{1} \in K_{1} \backslash K_{2}$. However, these laws are not compatible with the laws $(\sigma-1) w=0$, as this would imply that $a_{1}^{T} w=0$ which is falsified by the second observation. The procedure $P_{f}^{*}$ only accepts the most strongly corroborated laws, so that also in this case $\mathscr{B} \cap \mathscr{B}\left(a_{0}^{T}\right)$ is identified.

It is easily checked that for $\tau_{1}=\tau_{2}=1$ there holds $P_{f}^{*}(d)=\left(\mathbb{R}^{3}\right)^{\mathbb{Z}}$, that for $\tau_{2}=1$ and $\tau_{1} \geq 2$ there holds $P_{f}^{*}(d)=\mathscr{B}\left(a_{0}^{T}\right)$, that the same holds true for $\tau_{2} \geq 2$ and $\tau_{1}+\tau_{2} \leq 7$, and that in other 
cases $P_{f}^{*}(d)=\mathscr{B} \cap \mathscr{B}\left(a_{0}^{T}\right)$. In case of three observations there holds that generically $P_{f}^{*}(d)=\left(\mathbb{R}^{3}\right)^{\mathbf{Z}}$ if $\delta^{*}(T)=0$ and also if $\delta^{*}(T) \geq 1$ and $\tau_{3}=1$, and otherwise generically $P_{f}^{*}(d)=\mathscr{B}$. So the system is $(3, T)$ identifiable, provided that $\tau_{3}$ is sufficiently large.

Algorithm. An algorithm for computing the system identified by $P_{f}^{*}$ for general data is as follows. Assume without loss of generality that $T_{i}=\left[1, \tau_{i}\right], \quad i \in[1, N]$, and define the $q \times N$ matrix $W(t):=\left[w_{1}(t) \cdots w_{N}(t)\right], t \in T_{1}$, inserting * for unobserved elements. For $\delta \in\left[0, \delta^{*}(T)\right]$ define the $q(\delta+1) \times N\left(\tau_{1}-\delta\right)$ block-Hankel matrix $M_{\delta}$ by

$$
M_{\delta}=\left(\begin{array}{cccc}
W(1) & W(2) & \cdots & W\left(\tau_{1}-\delta\right) \\
W(2) & W(3) & \cdots & W\left(\tau_{1}-\delta+1\right) \\
\vdots & \vdots & & \vdots \\
W(\delta+1) & W(\delta+2) & \cdots & W\left(\tau_{1}\right)
\end{array}\right) .
$$

Let $H_{\delta}$ consist of those columns of $M_{\delta}$ which are fully observed, i.e. which contain no *. It is easily seen that $H_{\delta}$ has $C_{\delta}$ columns and that a law of degree $\delta$, say $r=\sum_{k=k_{0}}^{k_{0}+\delta} r_{k} s^{k} \in \mathbb{R}^{1 \times q}\left[s, s^{-1}\right]$ with $r_{k_{0}} \neq 0 \neq r_{k_{0}+\delta}$, is corroborated if and only if $\left(r_{k_{0}}, r_{k_{0}+1}, \ldots, r_{k_{0}+\delta}\right) \cdot H_{\delta}=0$.

The identified system $P_{f}^{*}(d)$ can be obtained as follows. Let $D_{\delta} \subset[1, q(\delta+1)]$ denote the set of indices of those rows in $H_{\delta}$ which are linearly dependent on the foregoing ones. Then the number $\delta^{*}$ in Definition 4.3 is given by $\delta^{*}=\max \left\{\delta \in\left[0, \delta^{*}(T)\right] ; D_{\delta} \cap[1, q \delta]=D_{\delta-1}\right\}$, i.e. incompatibility is obtained if there exists $i \in[1, q \delta]$ with $i \in D_{\delta} \backslash D_{\delta-1}$. The Hankel structure of $H_{\delta}$ implies that there exist a number $p \in[0, q]$, distinct indices $\left\{j_{1}, \ldots, j_{p}\right\} \subset[1, q]$, and numbers $\delta^{*} \geq v_{1} \geq \cdots \geq v_{p} \geq 0$, such that for $\delta \in\left[0, \delta^{*}\right]$ there holds $D_{\delta}=\{j \in[1, q(\delta+$ 1)]; $\left.j=j_{k}+n \cdot q, n \geq v_{k}, k \in[1, p]\right\}$. Then the identified system has $p$ outputs and $\left(v_{1}, \ldots, v_{p}\right)$ are its observability indices. An autoregressive representation is obtained as follows. Laws of degree $\delta:=v_{k}$ can be computed from the coefficients expressing the dependency of row $j_{k}+v_{k} q$ in $H_{\delta}$ on the foregoing rows of $H_{\delta}$. This is achieved, e.g. by determining a basis for the left kernel of the submatrix of $H_{\delta}$ obtained by deleting rows with index in $D_{\delta-1} \cup\left\{j_{k}+\delta q ; k \in\right.$ $[1, p]$ such that $\left.v_{k}<\delta\right\}$.

Similar algorithms are described in Willems $(1986 \mathrm{~b}, 1987)$ for the procedure $P^{*}$, when $T_{1}=\cdots=T_{N}=\mathbb{Z}$, and in Heij (1992) for the procedure $P_{1}^{*}$, when $N=1$.

\section{SYSTEM IDENTIFIABILITY}

In this section we describe identifiability of systems in $B$ on the basis of multiple observed finite time series by means of the procedure $P_{f}^{*}$ introduced in Section 4.

We use the following notation. Let the system $\mathscr{B} \in \mathbf{B}$ have identifiability index $I$ and let it have $p$ outputs and observability indices $v:=$ $\left(v_{1}, \ldots, v_{p}\right), \quad v_{1} \geq \cdots \geq v_{p} \geq 0 . \quad$ Let $\quad w_{i} \in \mathscr{B}$, $i \in[1, N]$, and let the most powerful unfalsified model for these data have $p_{\infty}$ outputs and observability indices $v_{\infty}:=\left(v_{1}^{\infty}, \ldots, v_{p_{\alpha}}^{\infty}\right)$. Suppose that the actually observed data consists of $d=\left\{\left.w_{i}\right|_{T_{i}} ; i \in[1, N]\right\}$, and as before assume that the observation intervals have lengths $\tau_{1} \geq \cdots \geq \tau_{N} \geq 1$. Let the identified model $P_{f}^{*}(d)$ have $p_{f}$ outputs and observability indices $v_{f}:=\left(v_{1}, \ldots, v_{p_{t}}^{f}\right)$. Define a partial ordering by $\left\{\left(v_{1}, \ldots, v_{p}\right) \leq\left(v_{1}^{\prime}, \ldots, v_{p}^{\prime}\right)\right\}: \Leftrightarrow\left\{v_{k} \leq v_{k}^{\prime}\right.$ for all $k \in[1, p]\}$.

Proposition 5.1. For every $\mathscr{B} \in \mathbb{B}$ the following holds $\mathscr{B}$-generically:

(i) for every $\delta \in\left[0, \delta^{*}(T)\right]$ the class of corroborated laws of degree $\delta$ for the observed data $d=\left\{\left.w_{i}\right|_{T} ; \quad i \in[1, N]\right\}$ coincides with the class of unfalsified laws of degree $\delta$ for $\left\{w_{1}, \ldots, w_{N_{\star}}\right\}$, where $N_{\delta}=\max \left\{i \in[1, N] ; \tau_{i} \geq \delta+1\right\}$

(ii) $p_{f}<p_{\infty}$, or $p_{f}=p_{\infty}$ and $v_{f} \leq v_{\infty}$;

(iii) if $N<I$ then $p_{\infty}=p, v_{\infty} \leq v$, and $v_{\infty} \neq v$ :

(iv) if $N \geq I$ and $\tau_{l} \geq\left[\frac{q}{I}+1\right]\left[v_{1}+1\right]-1$, then $p_{f}=p_{\infty}=p$ and $v_{f}=v_{\infty}=v$.

Proof. See Appendix.

The main identifiability result is expressed in the following theorem.

Theorem 5.2. A system $\mathscr{B} \in \mathbb{B}$ with identifiability index $I$ is $(N, T)$ identifiable by $P_{f}^{*}$ if and only if $N \geq I$ and $\tau_{l}$ is sufficiently large, with sufficient condition that $\tau_{l} \geq\left[\frac{q}{l}+1\right]\left[v_{1}+1\right]-1$.

\section{Proof. See Appendix.}

Corollary 5.3. A controllable system $\mathscr{B} \in \mathbb{B}$ is $(1, T)$ identifiable by $P_{f}^{*}$ if $\tau \geq\left(v_{1}+1\right)(q+1)-$ 1.

\section{Proof. See Appendix.}

It is intuitively evident that a system should be more easily identifiable if more observations from the system become available. In particular, if a system is $(N, T)$ identifiable then it should also be identifiable in case of additional information on $\left(N^{\prime}, T^{\prime}\right)$ with $N^{\prime} \geq N$ and with $T_{i}^{\prime} \supset T_{i}$ for all $i \in[1, N]$. We call a procedure 
conservative if it has this property for every system in $\mathbb{B}$.

Theorem 5.4. The procedure $P_{f}^{*}$ is conservative.

\section{Proof. See Appendix.}

Remarks. (i) The class of systems identifiable by $N$ finite time series coincides with that identifiable by $N$ infinite time series. In this sense there is no essential difference between the cases of infinite and finite time series. This is due to the completeness of the generating system. Indeed, systems which are not complete are not identifiable by finite time series. For example, observations on finite time intervals can not distinguish the incomplete system $l_{2}:=\{w \in$ $\left.\left(\mathbb{R}^{q}\right)^{\mathbb{Z}} ; \quad \sum_{t-\cdots x}^{x} \|\left. w(t)\right|^{2}<\infty\right\}$ from the system $\left(\mathbb{R}^{4}\right)^{7}$

(ii) Parameter identifiability is usually interpreted as a bijective correspondence between the parameters and the corresponding external behaviour. Without additional a priori information a similar bijective correspondence between observed time series and the underlying generating system can not be obtained. For example, the system $\left(\mathbb{R}^{q}\right)^{\mathbb{Z}}$ can never be definitely rejected as a candidate. A system can be generically uniquely identifiable in this stronger sense if the observability indices are known a priori.

(iii) The procedure $P_{f}^{*}$ could be slightly refined. For example, not only the degree of unfalsified laws but also the number of non-zero coefficients could be taken into account, cf. Heij (1988). Further, if the identified system has rank drop equal to the number of observations it could be argued that some more corroborated laws should be rejected, cf. Heij (1992).

(iv) One could obtain alternative procedures by simpler direct generalizations of the procedure $P_{1}^{*}$ for a single finite time series, as defined in Section 3. For observed data $d=\left\{\left.w_{i}\right|_{T_{i}} ; i \in\right.$ $[1, N]\}$ let $\mathscr{B}_{i}^{*}:=P_{1}\left(\left.w_{i}\right|_{r_{i}}\right)$ denote the system identified from the $i$ th observation. Define $P_{+}(d):=\sum_{i=1}^{N} \mathscr{B}_{i}^{*}$ as the procedure which only accepts laws that are corroborated by all observations, and let $P_{-}(d):=\bigcap_{i=1}^{N} \mathscr{B}_{i}^{*}$ be the procedure which accepts all laws that are corroborated by at least one observation. Further let $P_{N}^{*}(d):=\bigcap\{\mathscr{B}(r) ; r$ corroborated by $d$ ) provided that $d$ is compatible with this system, and let $P_{N}^{*}(d)=\left(\mathbb{P}^{q}\right)^{Z}$ otherwise. These procedures have unattractive properties. The procedures $P_{N}^{*}$ and $P_{+}$are not conservative. In particular, identifiability by $P_{N}^{*}$ can be obstructed by observing a given time series over a larger time interval, and identifiability by $P_{+}$can be obstructed by observing an additional time series on a small time interval. Finally, no system $\mathscr{B} \in \mathbb{B}$ with identifiability index $I>1$ is identifiable by $P_{-}$.

Next assume that all time series are observed on intervals of equal length. Consider data $d=\left\{\left.w_{i}\right|_{T_{i}} ; i \in[1, N]\right\}$ and $d^{\prime}=\left\{\left.w_{i}^{\prime}\right|_{\left.T_{i} ; i \in\left[1, N^{\prime}\right]\right\}}\right.$ observed from a system $\mathscr{B} \in \mathbb{B}$, and assume that $w_{i}=w_{i}^{\prime}$ for all $i \in[1, N] \cap\left[1, N^{\prime}\right]$ and $\tau^{\prime}:=\tau_{1}^{\prime}=$ $\tau_{2}^{\prime}=\cdots=\tau_{N}^{\prime}$. The following proposition expresses monotonicity properties of $P_{f}^{*}$ with respect to additional information.

Proposition 5.5. For every $: B \in \mathbb{B}$ the following holds $\mathscr{B}$-generically:

(i) if $N=N^{\prime}$ is fixed and $T_{i} \supset T_{i}^{\prime}$ for all $i \in[1, N]$, then $P_{f}^{*}(d) \subset P_{f}^{*}\left(d^{\prime}\right)$

(ii) if $N \geq N^{\prime}, T_{i}=T_{1}^{\prime}$ for all $i \in\left[1, N^{\prime}\right]$ and $\boldsymbol{\tau}=\boldsymbol{\tau}^{\prime}$ is fixed and sufficiently large, then $P_{f}^{*}(d) \supset P_{f}^{*}\left(d^{\prime}\right)$.

Proof. See Appendix.

The result in (i) means that for a fixed number of observed time series the identified system can generically be computed in a recursive way. On obtaining additional information the laws which were already identified are reconfirmed and it only remains to determine newly corroborated laws, i.e. those of maximal degree. Additional information of this kind leads to more restrictive systems. On the other hand, the result in (ii) shows that the identified system can get larger if an additional time series is observed. This is explained by the fact that the richness of the non-controllable part of the behaviour is only gradually detected when additional data become available.

To obtain system identifiability, a sufficient condition on the length of the observation intervals is given in Theorem 5.2. The next proposition provides a minimal bound in case all intervals have equal length $\tau:=\tau_{1}=\cdots=\tau_{N}$. It shows that the (largest) observability index of a system has an interpretation in terms of system observability in the sense of identifiability, and not only in classical terms of state observability.

Proposition 5.6.

(i) A system $\mathscr{B} \in \mathbb{B}$ is $(N, T)$ identifiable by $P_{f}^{*}$ if and only if $N \geq I(\mathscr{B})$ and $\tau \geq$ $v_{1}+\max \left\{1, \frac{q}{N}\left(v_{1}+1\right)\right\}$

(ii) in particular, if $N$ is sufficiently large then is identifiable if and only if $\tau \geq v_{1}+1$.

Proof. See Appendix. 
The results of this section show intrinsic limitations in the identifiability of noncontrollable systems. For instance, systems with identifiability index larger than one can not be recovered by a single experiment. In this case the data only provide partial information on the system behaviour.

\section{IDENTIFIABILITY FROM A PARTIAL IMPULSE RESPONSE}

In this section we consider modelling of a partially observed impulse response.

For every system $\mathscr{B} \in \mathbb{B}$ its $q$ variables $w$ can be partitioned into $m$ (causal) input variables, denoted by $u$, and $p:=q-m$ output variables, denoted by $y$. For a given system the numbers $m$ and $p$ are unique, but the partitioning of the system variables into inputs and outputs need not be unique, cf. Willems (1989). In the sequel we assume that $m \geq 1$ and $p \geq 1$, that the choice of inputs and outputs is fixed, and that $w=\left(\begin{array}{l}u \\ y\end{array}\right)$.

The impulse response of a system $\mathscr{B} \in \mathbb{B}$ with $m$ inputs is defined as the subset $\mathscr{B}_{I R} \subset \mathscr{B}^{m}$ given by $\mathscr{B}_{I R}:=\left\{I R=\left(w_{1}, \ldots, w_{m}\right) \in \mathscr{B}^{m} ; w_{i}=\left(\begin{array}{l}u_{i} \\ y_{i}\end{array}\right), u_{i}\right.$ is the $i$ th unit pulse on time $t=0, i \in[1, m]\}$. This set is an affine space of finite dimension, and it need not be a singleton. Usually a special element of $\mathscr{B}_{I R}$ is called "the" impulse response of $\mathscr{B}$, i.e. the unique element $I R_{c} \in \mathscr{B}_{\mathbb{R}}$ characterized by the fact that all outputs are zero in the past, i.e. $y_{i}(t)=0$ for $t<0, i \in[1, m]$. We call $I R_{c}$ the causal impulse response of $\mathscr{B}$. The sequence $\left\{\left[y_{1}(t) \cdots y_{m}(t)\right]\right.$, $t \geq 0\}$ corresponding to $I R_{c}$ consists of the Markov parameters of $\mathscr{B}$.

First we summarize some identifiability results for data consisting of a fully observed impulse response and for the procedure $P^{*}$ of most powerful unfalsified modelling, cf. Heij (1991). We call a subset $V \subset \mathscr{B}_{I R}$ of the impulse response generic if $\left(\mathscr{B}_{I R} \backslash V\right) \subset \bigcup\left\{p_{n}^{-1}(0) ; n \in \mathbb{N}\right\}$, where $p_{n}:\left(\left(\mathbb{R}^{q}\right)^{\mathbb{Z}}\right)^{m} \rightarrow \mathbb{R}$ are polynomials which are not identically zero on $\mathscr{B}_{I R}$.

\section{Theorem 6.1.}

(i) A system $\mathscr{B} \in \mathbb{B}$ is identifiable by the causal impulse response, i.e. $P^{*}\left(I R_{c}\right)=$ $\mathscr{B}$, if and only if $\mathscr{B}$ is controllable;

(ii) a system is identifiable by generic impulse responses, i.e. $P^{*}(I R)=\mathscr{B}$ for generic $I R \in \mathscr{B}_{I R}$, if and only if $m \geq I(\mathscr{B})$; (iii) systems with $m \geq p$ are identifiable from generic impulse response observations.

Observation of the causal impulse response is related to the minimal realization problem for controllable systems, cf. Kalman et al. (1969) and Silverman (1971). The theorem states that there are also non-controllable systems which are identifiable from (non-causal) impulse response observations.

Next we consider the case where an impulse response is only partially observed, i.e. on a finite time interval.

Example 6. Consider the system $\mathscr{B} \in \mathbb{B}$ with $m$ inputs and $p$ outputs related by $\sigma y_{1}=u_{1}$, $\sigma y_{i+1}=y_{i}, i \in[1, p-1]$. Let $e_{i}$ denote the $i$ th unit vector in $\mathbb{R}^{p}$ and let $E_{i}:=\left[\begin{array}{lll}e_{i} & 0 & \cdots\end{array}\right] \in \mathbb{R}^{p \times m}$ and $A:=\left[e_{2} \cdots e_{p}, 0\right] \in \mathbb{R}^{p \times p}$. Then $\mathscr{B}$ has autoregressive representation $\mathscr{B}:=\left\{\left(\begin{array}{l}u \\ y\end{array}\right) \epsilon\right.$ $\left.\left(\mathbb{R}^{m+p}\right)^{\mathbf{z}} ;(\sigma I-A) y-E_{1} u=0\right\}$. The system $\mathscr{B}$ is controllable and has observability indices $v_{1}=$ $\cdots=v_{p}=1$. The impulse response consists of the single element $I R_{c}$ with $I R_{c}(t)=E_{t}, t \in[1, p]$, and zero otherwise. The Hankel matrix corresponding to the observation $I R_{c}$ is given by

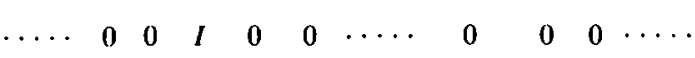

$$
\begin{aligned}
& \begin{array}{lllllllllllll}
\cdots & 0 & 0 & 0 & E_{1} & E_{2} & \cdots & E_{p-1} & E_{p} & 0 & \cdots
\end{array}
\end{aligned}
$$

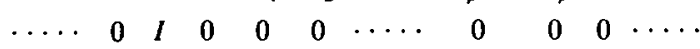

$$
\begin{aligned}
& \begin{array}{cccccccccccc}
\cdots & 0 & 0 & E_{1} & E_{2} & E_{3} & \cdots & E_{p} & 0 & 0 & \cdots
\end{array}
\end{aligned}
$$

Now suppose that $I R_{c}$ is observed on a finite interval $T \subset \mathbb{Z}$. We consider several cases. First suppose that we do not observe the past, so that $T=[0, \tau] . \quad$ If the system law $\sigma y_{1}=u_{1}$ is corroborated, then also the law $\sigma y_{1}+\sigma u_{1}=u_{1}$ is corroborated by the data, as $\left.\left(\sigma u_{1}\right)\right|_{T}=0$. This shows that the corroborated laws of degree 1 are incompatible with the data, as they would imply that $u_{1}=0$. Hence the system $\mathscr{B}$ is not identified by $P_{f}^{*}$ for any $\tau \in \mathbb{N}$, even if the condition of Proposition 5.6 on the length of the observation interval is satisfied. The same holds true for every observation interval $T \subset[0, \infty)$. This is caused by the fact that $P_{f}^{*}$ is a procedure for modelling arbitrary time series, while in this case the input is highly non-generic. Next suppose that $T \subset(-\infty, \tau]$ where the future observation interval $[1, \tau]$ satisfies the condition of Proposition 5.6, i.e. $\tau \geq 3+(2 p / m)$. If $\tau, m$ 
and $p$ are such that $3+(2 p / m) \leq \tau \leq p-1$, e.g. $\tau=4, m=10$ and $p=5$, then in the system identified by $P_{f}^{*}$ the $p$ th component of $y$ will be identically zero, so that $\mathscr{B}$ is not identified. This shows that the observation interval should contain a sufficiently long part of past and future.

From the Hankel matrix it is evident that $P_{f}^{*}\left(\left.I R\right|_{T}\right)=\mathscr{B}$, i.e. that $\mathscr{B}$ is identified from a partial impulse response, provided that $T \supset$ $[-1, p]$ and that $T$ has length at least $3+(2 p / m)$.

The following theorem states conditions for system identifiability on the basis of a partially observed impulse response. It is not assumed that there is any a priori information concerning the number or partitioning of input and output variables or concerning the number of state variables.

Theorem 6.2. A system $\mathscr{B} \in \mathbb{B}$ is identifiable by $P_{f}^{*}$ from an impulse response observation on the interval $T$, i.e. $P_{f}^{*}\left(\left.I R\right|_{T}\right)=\mathscr{B}$ for some $I R \in \mathscr{B}_{I K}$, if and only if $m \geq I(\mathscr{B})$ and $T$ extends sufficiently far into past and future. A sufficient condition is that $T=\left[-\tau_{1}, \tau_{2}\right]$, with $\tau_{2} \geq 2 n$ and $\tau_{1} \geq$ $\max \left\{\frac{p}{m}(n+1), 2 \tau_{2}\right\}$. In this case $\mathscr{B}$ is identified from generic partial impulse response observations on $T$.

\section{Proof. See Appendix.}

Remarks. (i) The definition of $P_{f}^{*}$ as a procedure for modelling arbitrary time series implies certain limitations for its application in case of particular data. Of special interest is the (classical) case of a partial observation of the causal impulse response on the interval $[0, \tau]$, i.e. starting at the moment of the impulse. It is clearly undesirable that $P_{f}^{*}$ also needs the zero past of this observation. If the input-output decomposition and the zero past are given $a$ priori, then the procedure $P_{f}^{*}$ can be used to identify an autonomous system for the output signals, i.e. for the Markov sequence. It can be shown that $\mathscr{B}$ is identifiable from a partial Markov sequence on the interval $[0, \tau]$ if and only if $\mathscr{B}$ is controllable, the output consists of the causal impulse response, and the observation interval is sufficiently large, e.g. $\tau \geq\left(\frac{p}{m}+1\right) \times$ $\left(v_{1}+1\right)$.

(ii) The minimal partial realization problem corresponds to a procedure $P_{\min }$ which determines controllable systems in $\mathbb{B}$ with smallest possible state dimension compatible with a partially observed causal impulse response, cf. Tether (1970), Kalman (1971) and Antoulas (1986). This procedure identifies systems of larger dimension if more observations become available. This seems somewhat unattractive, as additional information leads to less restrictive identified laws, i.e. to more indeterminateness of the system variables. On the other hand, the procedure $P_{f}^{*}$ determines the system in $\mathbb{B}$ which is optimally corroborated by the data, and Proposition 5.5 (i) shows that $P_{f}^{*}$ generically identifies more precise systems if the observation interval gets larger. This also holds true for partial impulse response observations from controllable systems in $\mathbb{B}$ with generic Markov parameters. The monotonicity property does not hold true for non-generic Markov parameters, like for the system $\mathscr{B}=\left\{\left(\begin{array}{l}u \\ y\end{array}\right) \in\left(\mathbb{R}^{2}\right)^{u} ; \sigma^{n} y=u\right\}$. If the impulse response is observed on $T=[-\tau, \tau]$, then $P_{f}^{*}$ identifies the system $\left\{\left(\begin{array}{l}u \\ y\end{array}\right) ; y=0\right\}$ if $\tau \leq n-1$. the system $\left(\mathbb{R}^{2}\right)^{\prime}$ if $n \leq \tau<\frac{1}{2}(3 n+1)$, and $B$ if $\tau \geq \frac{1}{2}(3 n+1)$.

\section{CONCLUSION}

This paper describes results on identifiability of linear, time invariant, complete systems from data consisting of a finite number of observed finite time series. For this purpose an identification procedure is presented that identifies the system which is optimally corroborated by the data. This procedure has attractive properties, and the identified system can be determined by an algorithm based on Hankel matrices defined in terms of the data. A complete result on identifiability is obtained. A specification is given of the minimally required number of observed time series and of the minimally required length of the observation intervals. The case of a partially observed impulse response is analysed in detail.

The results presented in this paper complete identifiability results for infinite time series or a single observed finite time series as presented in Heij $(1988,1992)$. The main distinction is that for multiple observed finite time series the collection of all corroborated laws is in general not compatible with the observed data. This problem is solved by accepting only the most strongly corroborated laws.

\section{REFERENCES}

Antoulas, A. C. (1986). On recursiveness and related topics in linear systems. IEEE, AC-31, 1121-1135.

Caines, P. E. (1988). Linear Stochastic Systems. Wiley, New York. 
Chen, C. T. (1987). Techniques for identification of linear time-invariant multivariable systems. In $C$. $T$. Leondes (Ed.), Control and Dynamic Systems, 26, 1-34. Academic Press, New York.

Fisher, F. M. (1966). The Identification Problem in Econometrics. McGraw-Hill, New York.

Gevers, M. and V. Wertz (1984). Uniquely identifiable state space and ARMA parametrizations for multivariable linear systems. Automatica, 20, 333-347.

Gevers, M. and V. Wertz (1987). Techniques for the selection of identifiable parametrizations for multivariable linear systems. In C. T. Leondes (Ed.), Control and Dynamic Systems, 26, 35-86. Academic Press, New York.

Grewal, M. S. and K. Glover (1976). Identifiability of linear and non-linear dynamical systems. IEEE, AC-21, 833-837.

Guidorzi, R. (1975). Canonical structures in the identification of multivariable systems. Automatica, 11, 361-374.

Guidorzi, R. (1981). Invariants and canonical forms for systems structural and parametric identification. Automatica, 17, 117-133.

Hannan, E. J. and M. Deistler (1988). The Statistical Theory of Linear Systems. Wiley, New York.

Heij, C. (1988). Exact modeling of a finite time series. SIAM J. Contr. Opt., 26, 83-111.

Heij, C. (1991). Identification of non-controllable systems from impulse response measurements. Report 9120/A, Econometric Institute, Rotterdam, The Netherlands.

Heij, C. (1992). Exact modelling and identifiability of linear systems. Automatica, 28, 325-344.

Hsiao, C. (1983). Identification. In Z. Griliches and M. D. Intriligator (Eds), Handbook of Econometrics, 1, 223-283. North-Holland, Amsterdam.

Kalman, R. E. (1971). On minimal partial realizations of a linear input/output map. In R. E. Kalman and N. De Claris (Eds), Aspects of Network and Systems Theory, 385-407. Holt, Rinehart and Winston, New York.

Kalman, R. E. (1983). Identifiability and modelling in econometrics. In P. R. Krishnaiah (Ed), Developments in Statistics, 4, 97-136. Academic Press, New York.

Kalman, R. E., P. L. Falb and M. A. Arbib (1969). Topics in Mathematical System Theory. McGraw-Hill, New York.

Ljung, L. (1987). System Identification: Theory for the User. Prentice-Hall, Englewood Cliffs, NJ.

Silverman, L. M. (1971). Realization of linear dynamical systems. IEEE, AC-16, 554-567.

Sontag, E. D. (1979). On the observability of polynomial systems, I: finite-time problems. SIAM J. Contr. Opt., 17, 139-151.

Sontag, E. D. (1980). On the length of inputs necessary to identify a deterministic linear system. IEEE, AC-25, 120-121.

Tether, A. J. (1970). Construction of minimal linear state-variable models from finite input-output data IEEE, AC-15, 427-436.

Willems, J. C. (1986a). From time series to linear system, part I: Finite dimensional linear time invariant systems. Automatica, 22, 561-580.

Willems, J. C. (1986b). From time series to linear system part II; Exact modelling. Automatica, 22, 675-694.

Willems, J. C. (1987). From time series to linear system, part III: Approximate modelling. Automatica, 22, 87-115.

Willems, J. C. (1989). Models for dynamics. In U. Kirchgraber and H. O. Walther (Eds), Dynamics Reported, 2, 171-269. Wiley, New York.

Willems, J. C. (1991). Paradigms and puzzles in the theory of dynamical systems. IEEE, AC-36, 259-294.

\section{APPENDIX: PROOFS}

Proof of Proposition 4.1. For (ii) a simple example is given in the text. Similar results hold true for systems with identifiability index $I(\mathscr{B}) \geq 2$.

In order to show (i) define the $q \times N$ matrix $W(t):=$ $\left[w_{1}(t) \cdots w_{N}(t)\right], t \in \mathbb{Z}$, inserting * for unobserved elements.
For $\delta \in \mathbf{N} \cup\{0\}$ define the block-Hankel matrix $M_{\delta}$ by

$M_{\mathrm{\delta}}=\left(\begin{array}{ccccc}\cdots & W(t-1) & W(t) & W(t+1) & \cdots \\ \cdots & W(t) & W(t+1) & W(t+2) & \cdots \\ & \vdots & \vdots & \vdots & \\ \cdots & W(t+\delta-1) & W(t+\delta) & W(t+\delta+1) & \cdots\end{array}\right)$

Further let $H_{\delta}$ consist of those columns of $M_{\delta}$ which are fully observed, i.e. which contain no *. So $H_{\delta}$ has $q(\delta+1)$ rows and $\sum_{i=1}^{N}\left[\tau_{i}-\delta\right]^{+}$columns.

It is easily verified that a law of degree $\delta$ is unfalsified by the data if and only if it, considered as a $1 \times q(\delta+1)$ real vector, belongs to the left kernel of $H_{0}$. For $\delta>\delta^{*}(T)$ this kernel is always non-zero, so that no law of such degree is corroborated. It remains to prove that for $\delta \leq \delta^{*}(T)$ there holds that generically $\operatorname{rank}\left(H_{\delta}\right)=q(\delta+1)$. Define the polynomial in the data $p(d):=\operatorname{det}\left(H_{\delta}^{\prime}\right)$, where $H_{\delta}^{\prime}$ is the square submatrix of $H_{\delta}$ consisting of its first $q(\delta+1)$ columns. Taking into account the Hankel structure of $H_{\delta}^{\prime}$ it follows that the polynomial term obtained by the product of the elements on the diagonal of $H_{0}^{\prime}$ has coefficient +1 in $p$. So for $\delta \leq \delta^{*}(T)$ the matrix $H_{\delta}$ generically has full row rank.

Proof of Proposition 5.1. The proof of these crucial results is rather technical and lengthy. We prove the parts of the proposition in the order (i), (iii), (ii), (iv)

(i) Let $\mathscr{B} \in \mathbb{B}, N \in \mathbb{N}$ and $T=\left\{T_{1}, \ldots, T_{N}\right\}$ be fixed. For simplicity of notation we assume that $T_{i}=\left[1, \tau_{i}\right], i \in[1, N]$. For $w_{i} \in G, i \in[1, N]$, and $\delta \geq 0$ define $W_{\gamma} \in\left(R^{a \times N_{A}}\right)^{\prime}$ by $W_{\delta}(t):=\left[w_{1}(t) \cdots w_{N_{\delta}}(t)\right], \quad t \in \mathbb{Z}$, and define $H_{\delta}\left(W_{\delta}\right) \in$ $\mathbf{R}^{a(\delta+1) \times \gamma}$ by $H_{\delta}\left(W_{\delta}\right):=\operatorname{col}\left(W_{\delta}, \sigma W_{\delta}, \ldots, \sigma^{\delta} W_{\delta}\right)$. Then for observed data $d=\left\{\left.w_{i}\right|_{T} ; i \in[1, N]\right\}$ the submatrix $H_{\delta} \in \mathbf{R}^{q(\delta+1) \times c_{\delta}}$, defined in the proof of Proposition 4.1, consists of those columns of $H_{b}\left(W_{\delta}\right)$ which are fully observed. Define the set of dependency indices $D_{\delta}\left(W_{\delta}\right)$ and $D_{\delta}(d)$ as the set of indices of those rows in $H_{\delta}\left(W_{\delta}\right)$ and $H_{\delta}(d)$ respectively which are linearly dependent on the foregoing ones. Finally let $H_{\delta}^{\text {red }}\left(W_{\delta}\right)$ and $H_{\delta}^{\text {red }}(d)$ denote the matrices obtained from respectively $H_{\delta}\left(W_{\delta}\right)$ and $H_{s}(d)$ by deleting the rows with index in respectively $D_{\delta}\left(W_{\delta}\right)$ and $D_{\delta}(d)$.

We will show that generically for all $\delta \in\left[0, \delta^{*}(T)\right]$ there holds $D_{\delta}(d)=D_{\delta}\left(W_{\delta}\right)$ if $d=\left\{\left.w_{i}\right|_{T_{i}} ; i \in[1, N]\right\}$. Assuming for the moment that this holds true, (i) is shown as follows. Clearly any unfalsified law $r$ of degree $\delta \leq \delta^{*}(T)$ for $W_{\delta}$ is a corroborated law for the data $d$, as in particular

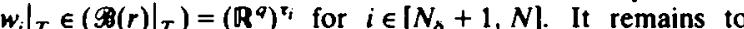
show that every law $r$ of degree $\delta \leq \delta^{*}(T)$ which is corroborated by $d$ is also unfalsified by $W_{A}$. Such a law $r$ corresponds to dependency of a row with index $j \in[q \delta+$ $1, q(\delta+1)]$ of $H_{\delta}(d)$, denoted by $\rho_{j}(d)$, on the foregoing rows. Then $\rho_{l}(d)=a H_{s}^{\text {red }}(d)$ expresses the law $r$ for some appropriate rowvector $a$. As $j \in D_{\delta}(d)$ there holds by assumption that $j \in D_{\delta}\left(W_{\delta}\right)$. In this case the $j$ th row of $H_{\delta}\left(W_{\delta}\right)$, denoted by $\rho_{i}\left(W_{\delta}\right)$, can be expressed as $\rho_{i}\left(W_{\delta}\right)=a^{\prime} H_{\delta}^{\text {red }}\left(W_{\delta}\right)$. This implies that in particular $\rho_{j}(d)=$ $a^{\prime} H_{\delta}^{\text {red }}(d)$, and as $H_{\delta}^{\text {red }}(d)$ has full row rank it follows that $a^{\prime}=a$. This shows that the law $r$ is also unfalsified by $W_{\partial}$.

Finally we show that for $\delta \leq \delta^{*}(T)$ generically $D_{\delta}(d)=$ $D_{\delta}\left(W_{\delta}\right)$. The inclusion $D_{\delta}\left(W_{\delta}\right) \subset D_{\delta}(d)$ is trivial, so it remains to show that generically $D_{\delta}(d) \subset D_{\diamond}\left(W_{\diamond}\right)$. If this would not hold true then there would exist an index $j \in[1, q(\delta+1)]$ such that $j \in D_{\delta}(d) \backslash D_{\delta}\left(W_{\delta}\right)$ is not generically false. Let $j_{0}$ be the smallest index with this property, let $W_{b}$ be such that $j_{0} \in D_{\delta}(d) \backslash D_{\delta}\left(W_{\delta}\right)$ and let $H_{\text {sub }}(d)$ and $H_{\text {sub }}\left(W_{\delta}\right)$ denote the submatrices of respectively $H_{\delta}(d)$ and $H_{\delta}\left(W_{\delta}\right)$ consisting of the rows with index in $\left[1, j_{0}\right] \backslash D_{\delta}\left(W_{\delta}\right)$, i.e. including $j_{0}$. Denote the number of rows of $H_{\text {sub }}(d)$ and $H_{\text {uub }}\left(W_{\theta}\right)$ by $n$. The foregoing would imply that rank $\left(H_{\text {sub }}(d)\right)<n$ is not generically false, and hence rank $\left(H_{\text {aub }}(d)\right) \leq n-1$ for all data $d$. As $\delta \leq \delta^{*}(T)$ there holds that the number of columns of $H_{\text {sut }}(d)$ is $C_{\delta} \geq q(\delta+1) \geq n$. Hence there exists a column with index $m \leq n$ of $H_{\text {sub }}(d)$ which is generically linearly dependent on the foregoing ones.

It is simple matter of exploiting the Hankel structure of 
$H_{\text {sut }}(d)$ to show that generic dependence of one columin in $H_{\text {sut }}(d)$ on the foregoing ones implies that generically also all its next columns are linearly dependent on the foregoing ones. This simply requires a reordering of the numbering of the $N$ observations, in case the next column concerns a nex time series on the same interval $[t, t+\delta]$ as the current column, or a relabelling of time, in case the next column concerns a time series on a next time interval $\mid t+1, t+\delta+$ 1). This implies in particular that generically $\operatorname{rank}\left(H_{\text {sub }}\left(W_{o}\right)\right) \leq m-1 \leq n-1$, contradicting the fact that $H_{\text {sub }}\left(W_{0}\right)$ has full rank as its rows correspond to indices in $[1, q(\delta+1)] \backslash D_{\partial}\left(W_{\partial}\right)$. This contradiction shows that generically an index $j_{01}$ as indicated does not exist, i.e. that generically $D_{\lambda}(d) \subset D_{\lambda}\left(W_{\partial}\right)$.

(iii) Let the components of $w$ be ordered such that $w=$ $\left(\begin{array}{l}u \\ y\end{array}\right)$, where $u$ consists of $m$ input variables and $y$ of $p$ output variables of $2 B$. Let $W:=\left(w_{1}, \ldots, w_{N}\right) \in A^{N}$ and let $d=\left\{w_{i} \mid \tau ; i \in[1, N]\right\}$

As all laws of $\rightarrow$ are unfalsified by $W$, it follows that $P^{*}(W):=\bigcap\{\mathscr{B}(r) ; r$ unfalsified by $W\} \subset \mathscr{B}$. This implies that $p_{x} \geq p$ and that $v_{k}^{x} \leq v_{k}$ for all $k \in[1, p]$. As $u$ is free in $\mathscr{B}$. i.e. for every $u \in\left(\mathbb{R}^{m}\right)^{7}$ there exist $y \in\left(\mathbb{R}^{p}\right)^{g}$ with $\left(\begin{array}{l}u \\ y\end{array}\right) \in \mathscr{B}$, it follows from Proposition 3.1 that generically the matrix in $\mathbb{R}^{m(\partial+1) \times \pi}$ consisting of the input rows of $H_{\delta}(W):=\operatorname{col}\left(W, \ldots \sigma^{\lambda} W\right)$ has rank $m(\delta+1)$. Hence

generically $q-p_{x}=\lim _{\lambda \rightarrow \infty} \delta^{-1} \operatorname{rank}\left(H_{\lambda}(W)\right) \geq m$, i.e. $p_{x} \leq p$ We conclude that generically $p_{x}=p$ and $v_{x} \leq v$. It remains to show that $v_{x} \neq v$. It is easily shown that for every $\mathscr{B}^{\prime} \in \mathbb{B}$ there holds $\operatorname{dim}\left(\left.\dot{B}^{\prime}\right|_{(1, r)}\right)=m^{\prime} \tau+\sum_{i-1}^{p} v_{1}\left(B^{\prime}\right)$ for all $\tau \geq$ $v_{1}\left(\mathscr{B}^{\prime}\right)$. If $v_{x}=v$, then $P^{*}(W) \subset \mathscr{B}$ and $\operatorname{dim}\left(\left.P^{*}(W)\right|_{\mid 1, r]}\right)=$ $\operatorname{dim}\left(\left.\mathscr{B}\right|_{|1, r|}\right)$ would imply that $\left.P^{*}(W)\right|_{|1, \tau|}=\left.\mathscr{B}\right|_{|1, \tau|}$ for all $\tau \geq v_{1}$. Shift invariance and completeness imply that $P^{*}(W)=\mathscr{B}$. It is shown in Heij (1992, Proposition 22(ii)) that for $N<I(98)$ always $P^{*}(W) \neq \mathscr{B}$. Hence it follows that $v_{\infty} \neq v$

(ii) It follows from (iii), for $N<1$, and from Theorem 3.2 , for $N \geq I$, that generically $p_{x}=p$. Hence generically no law on the input variables is unfalsified for $w_{\diamond}=\left(w_{1}, \ldots, w_{v_{A}}\right)$ i.e. generically $j+n q \notin D_{\diamond}\left(W_{\diamond}\right)$ for every $j \in[1, m], n \geq 0$, $\delta \geq 0$. The proof of (i) shows that hence generically $j+n q \notin D_{\delta}(d)$ for every $j \in[1, m], \quad n \geq 0, \delta \in\left[0, \delta^{*}(T)\right]$. This means that the variables $u$ are also input variable in $P_{f}^{*}(d)$, hence $p_{f} \leq p$. Suppose that $p_{f}=p$, then it remains to show that $v_{k} \leq v_{k}^{x}$ for all $k \in[1, p]$. If $p_{f}=p$ then for every output variable $y_{k}, k \in[1, p], P^{*}$ accepts the corroborated law of least degree $v_{k}^{f}$. There holds that either $v_{k}^{J} \leq \delta^{*}(T)<$ $v_{k}^{*}$ or $v_{k}^{x} \leq \delta^{*}(T)$. In the last case there evidently is a corroborated law of degree $v_{k}^{*}$ for data $d$, hence $v_{k}^{\prime} \leq v_{k}^{*}$

(iv) Theorem 3.2 implies that generically $p_{x}=p$ and $v_{x}=v$, and (ii) implies that it suffices to show that $p_{f} \geq p$ and $v_{f} \geq v$. The condition $r_{1} \geq\left(\frac{q}{l}+1\right)\left(v_{1}+1\right)-1$ implies that $\delta^{*}(T) \geq v_{1}$, and as $I(B) \leq p \leq q$ it follows that $N_{\delta} \geq I$ for all $\delta \in\left[0, v_{1}\right]$. The result in (i) shows that generically every corroborated law of degree $\delta \leqslant v_{1}$ for data $d$ is an unfalsified law for $w_{b}=\left(w_{1}, \ldots, w_{N_{b}}\right)$, and as $N_{0} \geq I$ Theorem 3.2 implies that generically this is a law of $\$ 0$. So generically the class of all corroborated laws of degree at most $v_{1}$ is compatible with the data $d$. Definition 4.3 implies that hence generically $P_{f}^{*}(d) \subset C B$, so $p_{f} \geq p$. As moreover every corroborated law of degree $\delta \leq v_{1}$ is generically a law of $\mathscr{B}$ it follows that $v_{k}<v_{k}^{f}, k \in[1, p]$

Proof of Theorem 5.2. If $N<I$ then Proposituon 5.1 (ii) and (iii) imply that generically $p_{f}<p$ or $v_{f} \neq v$, so $P_{f}^{*}(d) \neq B$ for generic data. Hence identifiability requires that $N \geq I$. Moreover, $t_{\text {, }}$ should be sufficiently large, as for laws of degree $\delta \geq \tau$, the observations $i \in[I, N]$ provide no additional corroboration. For $N \geq I$ and $\tau_{1} \geq\left(\frac{q}{l}+1\right)\left(v_{1}+\right.$ 1) -1 it follows from Proposition 5.1 (iv) that generically $p_{f}=p$ and $v_{f}=v$. The proof of Proposition 5.1 (iv) moreover shows that gencrically $P_{f}^{*}(d) \subset \mathscr{A}$. The reasoning given in the last part of the proof of Proposition 5.1 (iii) shows that then $P_{f}^{*}(d)=: \not h$

Proof of Corollary 5.3. This follows from Theorem 5.2, as for a controllable system $\mathscr{B}=\mathscr{B}(R)$ rank $\left(R\left(s, s^{\prime}\right)\right)$ is constant over $\mathbb{C} \backslash\{0\}$, so that $I(B B)=1$.

Proof of Theorem 5.4. Suppose that $\mathbb{B} \in \mathbb{B}$ is $(N, T)$ identifiable by $P_{f}^{*}$ and let $\left(N^{\prime}, T^{\prime}\right) \geq(N, T)$. Let $d^{\prime}:=$ $\left\{\left.w_{i}\right|_{T} ; i \in\left[1, N^{\prime}\right]\right\}$ and define $d:=\left\{\left.w_{i}\right|_{T} ; i \in[1, N]\right\}$. Accord ing to Theorem 5.2 there holds $N \geq \xi$, and as $P_{f}^{*}(d)=\mathbb{B}$ generically there holds $\delta^{*}(T) \geq v_{1}$. As $\left(N^{\prime}, T^{\prime}\right) \geq(N, T)$ it follows that $N^{\prime} \geq N \geq I, \quad \delta^{*}\left(T^{\prime}\right) \geq v_{1}, \tau_{i}^{\prime} \geq \tau_{i}$ for $i \in[1, N]$, and hence $N_{o}^{\prime} \geq N_{\delta}$ for all $\delta \leq \delta^{*}(T)$. Proposition 5.1 (i) states that generically every law of degree $\delta \leq \delta^{*}(T)$ which is corroborated by $d^{\prime}$ is also unfalsified for $\left(w_{1}, \ldots, w_{N_{i}}\right)$ hence also for $\left(w_{1}, \ldots, w_{N_{\lambda}}\right)$, and then it is corroborated by $d$, i.e. generically it is a law of $\mathscr{B}$. This implies that generically $P_{f}^{\prime \prime}\left(d^{\prime}\right) \subset \mathscr{B}$, so $p_{f}^{\prime} \geq p$ and $v_{f}^{\prime} \leq v$. Proposition 5.1 (ii) and Theorem 3.2 imply that hence $p_{f}^{\prime}=p$. As generically every law of degree $\delta \leq \delta^{*}(T)$ which is corroborated by $d^{\prime}$ is also corroborated by $d$ it follows that generically $v=v_{f} \leq v_{f}^{\prime}$. Hence $P_{f}^{*}\left(d^{\prime}\right) \subset \mathscr{B}, p_{f}^{\prime}=p$ and $v_{f}^{\prime}=v$. The reasoning given in the last part of the proof of Proposition 5.1 (iii) shows that then generically $P_{f}^{*}\left(d^{\prime}\right)=\mathscr{B}$, i.c. $\mathscr{B}$ is $\left(N^{\prime}, T^{\prime}\right)$ identifiable by $P^{*}$

Proof of Proposition 5.5. For all $\delta \leq \delta^{*}\left(T^{\prime}\right) \leq \delta^{*}(T)$ there holds $N_{\delta}^{\prime}=N_{\delta}=N$, and Proposition 5.1 (i) implies that generically the class of corroborated laws of degree at most $\delta$ for $d$ coincides with that class for $d^{\prime}$. This holds true especially for $\delta:=v_{1}\left(P_{f}^{*}\left(d^{\prime}\right)\right)$, and Definition 4.3 implies that then $P_{f}^{*}(d) \subset P_{f}^{*}\left(d^{\prime}\right)$. This proves (i).

In order to prove (ii), let $N \geq N^{\prime}$ and $W^{\prime}=\left(w_{1}, \ldots, w_{N}^{\prime}\right)$ $W=\left(w_{1}, \ldots, w_{N}\right)$. All unfalsified laws for $W$ are clearly unfaisified for $W^{\prime}$, so $P^{*}(W) \supset P^{*}\left(W^{\prime}\right)$. It suffices to show that for $t$ sufficiently large there holds generically that $P_{f}^{*}(d)=P^{*}(W)$. We show that this holds true for $\tau \geq v_{1}+\max \left\{1, \frac{q}{N}\left(v_{1}+1\right)\right\}$. Indeed, in this case $\delta^{*}(T) \geq$ $v_{1}$. for all $\delta \leq \delta^{*}(T)$ there holds $N_{\partial}=N$, and Proposition 5.1 (i) and Definition 4.3 imply that generically $P_{f}^{*}(d)=$ $P^{*}(W)$.

Proof of Proposition 5.6. Identifiability requires $N \geq I$ and $\delta^{*}(T) \geq v_{1}$, i.e. $\tau \geq v_{1}+\max \left\{1, \frac{q}{N}\left(v_{1}+1\right)\right\}$. That these are also sufficient conditions follows from the proof of Proposition 5.5 (ii) and from Theorem 3.2. This shows (i) and (ii) is an immediate corollary.

Proof of Theorem 6.2. Denote the observation interval by $T=\left[-\tau_{1}, t_{2}\right]$. If $\tau_{1}<0$ or $\tau_{2}<0$ then evidently the inpu variables $u$ are modelled by $P_{f}^{*}$ as being identically zero. Hence identifiability requires that $\tau_{1} \geq 0$ and $\tau_{2} \geq 0$, and $\tau_{1}$ and $\tau_{2}$ should be sufficiently large. Further, if $m<I(B)$ then $\mathscr{B}$ is not identifiable by any partial impulse response. Indeed. using the notation of Proposition 5.1, if the number and the components of the output variables are correctly identified. i.e. $p_{f}=p$, then the reasoning given in the last part of the proof of Proposition 5.1 (ii) shows that $v_{f} \leq v_{x}$. Moreover, as evidently $p_{x}=p$ for every $I R \in \phi_{I R}$, the reasoning given in the last part of the proof of Proposition 5.1 (iii) shows that $v_{x} \leq v$ and $v_{\cdot x} \neq v$. We conclude that for $m<I(S B)$ either the outputs are not correctly identified or $v_{f} \neq v$, hence in this case $\Rightarrow$ is not identifiable by $P_{f}^{*}$ for any partial impulse response observation. 
Next we show that $\mathscr{B}$ is identifiable from generic partial impulse response observations if $m \geq I(\mathscr{B})$ and the observation interval $T$ satisfies the conditions $r_{2} \geqq 2 n$ and $\tau_{1} \geq \max \left\{\frac{p}{m}(n+1), 2 \tau_{2}\right\}$. It is straightforward to show that these conditions imply that $\delta^{*}(T) \geq n \geq v_{1}$. Using the notation of the proof of Proposition 5.1 we state an auxiliary lemma.

\section{Lemma}

(i) There exist no corroborated laws for the inputs;

(ii) for every $I R \in \mathscr{B}_{I R}$ and all $\delta \leq v_{1}$ there holds $D_{\delta}\left(\left.I R\right|_{T}\right)=D_{\delta}(I R)$.

Supposing for the moment that this lemma holds true, the theorem is shown as follows. The reasoning in the proof of Proposition 5.1 (i) shows that (ii) implies that every corroborated law of degree $\delta \leq v_{1}$ for the partially observed impulse response is also unfalsified for the full response $I R$. Hence in particular the collection of all corroborated laws of degree $\delta \leq v_{1}$ is compatible with $\left.I R\right|_{T}$, and especially the laws of a minimal representation of $\mathscr{P}$ are corroborated and compatible. so $P_{f}^{*}\left(\left.I R\right|_{T}\right) \subset \mathscr{B}$. Morenver, (i) implies that $p_{f}=p_{\infty}$, and (ii) that $v_{f}=v_{\infty}$. The reasoning given in the last part of the proof of Proposition 5.1 (iii) shows that then $P^{*}(I R)=P_{f}^{*}\left(\left.I R\right|_{T}\right)$. According to Theorem 6.1 (ii) the fact that $m \geq I(\mathscr{B})$ implies that generically $P^{*}(I R)=\mathscr{B}$ and hence also $P_{f}^{*}\left(\left.I R\right|_{r}\right)=\mathscr{B}$, which proves the theorem.

It remains to prove the lemma. If the system $S$ is memoryless, i.e. the minimal number of state variables is $n=0$, then this result is trivial. So suppose that $n \geq 1$, and let $(A, B, C, D)$ be a minimal realization of $\mathscr{B}$, i.e. $\mathscr{B}=$ $\left\{\left(\begin{array}{l}u \\ y\end{array}\right) \in\left(\mathbb{R}^{m} \times \mathbb{R}^{p}\right)^{\boldsymbol{Z}} ; \exists x \in\left(\mathbb{R}^{n}\right)^{\boldsymbol{Z}}\right.$ with $\left.\left(\begin{array}{c}\sigma x \\ y\end{array}\right)=\left(\begin{array}{ll}A & B \\ C & D\end{array}\right)\left(\begin{array}{l}x \\ u\end{array}\right)\right\}$ with $n:=\sum_{k=1}^{p} v_{k}$, cf. Willems (1986, Theorem 6). Define the subspace $\mathscr{X}_{0} \subset \mathbb{R}^{n}$ by $\mathscr{X}_{0}:=\bigcap_{k \geq 0}$ im $\left(A^{k}\right)$, then the inverse of $\left.A\right|_{x_{0}}$ exists and will be denoted by $A$... It is easily seen that $B_{I R}$ is parametrized by arbitrary initial conditions $X_{0}:=\left(x_{1}(0), \ldots, x_{m}(0)\right) \in \mathscr{Z}_{0}^{m}$ such that $Y(t)=C A^{t} X_{0}$ for $t<0, \quad Y(0)=C X_{0}+D$, and $Y(t)=C A^{t-1}\left(B+A X_{0}\right)$ for $t>0$. As $X_{0}$ is a polynomial function of $\left.I R\right|_{\{-n,-1\}}$ it suffices to prove that the lemma holds true for generic $X_{0} \in \mathscr{X}_{0}^{m}$. Using the notation of the proof of Proposition 4.1 and defining $X_{1}:=B+A X_{0}$, it follows that for $\delta \leq \delta^{*}(T)$ the matrix $H_{\delta}$ is given by

$$
\begin{array}{ccccccccc}
0 & \cdots & 0 & 0 & \cdots & I & 0 & \cdots & 0 \\
C A_{-}^{\tau_{1} X_{0}} & \cdots & C A_{-}^{\delta+1} X_{0} & C A^{\delta} X_{0} & \cdots & C X_{0}+D & C X_{1} & \cdots & C A^{\tau_{2}-1-\delta} X_{1} \\
0 & \cdots & 0 & 0 & \cdots & 0 & 0 & \cdots & 0 \\
C H_{0}^{\tau_{1}-1} X_{0} & \cdots & C A_{-}^{\delta} X_{0} & C A_{-}^{\delta-1} X_{0} & \cdots & C X_{1} & C A X_{1} & \cdots & C A^{\tau_{2}-\delta} X_{1} \\
\vdots & & \vdots & \vdots & & \vdots & \vdots & & \vdots \\
0 & \cdots & 0 & i & \cdots & 0 & 0 & \cdots & 0 \\
C A^{\tau_{1}-\delta} X_{0} & \cdots & C A_{-} X_{0} & C X_{0}+D & \cdots & C A^{\delta-1} X_{1} & C A^{\delta} X_{1} & \cdots & C A^{\tau_{2}-1} X_{1}
\end{array}
$$

Denote $H_{\delta}:=\left[H_{1}, H_{2}, H_{3}\right]$ with $H_{1} \in \mathbb{R}^{q(\delta+1) \times m\left(r_{1}-\delta\right)}, H_{2} \in$ $\mathbb{R}^{q(\delta+1) \times m(\delta+1)}$ and $H_{3} \in \mathbb{R}^{q(\delta+1) \times m\left(x_{2}-\delta\right)}$.

We first show part (i) of the lemma. Let $\delta \leq \delta^{\bullet}(T)$ and suppose that $j \in D_{\partial}\left(\left.I R\right|_{T}\right)$ concerns an input variable. It is a matter of simple calculation to show that the conditions on the observation interval $T$ imply that $\tau_{1}-\delta^{*}(T) \geq n$, so that $H_{1}$ contains at least $n m$-block columns. Let $Z_{0}:=A^{t_{1}} X_{0}$, then an input dependency would mean that there exist vectors $a$ and $b$, with $0 \neq b \in \mathbb{R}^{m}$, such that $a^{T}\left[\operatorname{col}\left(C, C A, \ldots, C A^{\delta}\right)\right] \cdot\left[Z_{0}, A Z_{0}, \ldots, A^{\tau_{1}-\delta-1} Z_{0}\right.$, $\left.A^{\tau_{1}-\delta} Z_{n}\right]=\left(0, b^{T}\right)$. This is not possible as $\tau_{1}-\delta \geq n$.

In order to show part (ii) of the lemma, it suffices to consider dependencies concerning output variables. Suppose that $\delta \leq v_{1}$ and that $j \in D_{\delta}\left(\left.I R\right|_{T}\right)$ is a dependency concerning an output variable. The conditions on the observation interval $T$ imply that $\tau_{i} \geq v_{1}+n, i=1,2$, so that both $H_{1}$ and $H_{3}$ have at least $n$ m-block columns. Taking account of the Hankel structure of $H_{1}$ and $H_{3}$ it follows that the same dependency is obtained if $\tau_{1} \rightarrow \infty$ and $\tau_{2} \rightarrow \infty$, so that $j \in D_{\delta}(I R)$. This shows that for $\delta \leq v_{1}$ there holds $D_{\delta}\left(\left.I R\right|_{T}\right) \subset D_{\delta}(I R)$. As evidently $D_{\delta}(I R) \subset D_{\delta}\left(\left.I R\right|_{T}\right)$ this proves part (ii) of the lemma. 